\title{
Mixing and spectral multiplicity
}

\author{
E. ARTHUR ROBINSON, JR. \\ Department of Mathematics, University of Pennsylvania, Philadelphia, PA 19104, USA
}

(Received 12 November 1984 and revised 15 April 1985)

Abstract. A mixing transformation is constructed which has non-simple spectrum of finite multiplicity. This example is based on a rank 1 mixing transformation and is constructed by cutting and stacking. It can be made to be mixing of all orders.

\section{Introduction}

Let $T$ be an invertible ergodic measure preserving transformation of a Lebesgue probability space $(X, \mu)$, and let $U_{T}$ be the induced unitary operator on the complex Hilbert space $L^{2}(X, \mu) ; U_{T} f(x)=f(T x)$. The spectral multiplicity $M_{T}$ of $T$ is the maximal spectral multiplicity of $U_{T}$ as defined in terms of the spectral theorem (cf. [10]). In this paper we consider the question of what values of $M_{T}$ are possible for mixing transformations $T$.

It was proved some time ago by Oseledec [13] that non-simple spectrum of finite multiplicity (i.e. $1<M_{T}<\infty$ ) occurs for certain weak mixing transformations $T$. Later, the methods of Oseledec were refined in [15] to obtain, for any given positive integer $m$, a weak mixing transformation $T$ with $M_{T}=m$. Since then, other techniques have appeared for constructing different sorts of transformations with non-simple spectrum of finite multiplicity (cf. [10], [16], [17], [6], [11]). Without going into details we note that each of these fails in some fundamental way to be mixing.

Many mixing transformations have countable Lebesgue spectrum and therefore do not have finite multiplicity. No transformation with positive entropy can have finite multiplicity. On the other hand, there is a well known example of a mixing transformation with simple spectrum; the Ornstein rank 1 mixing transformation. In the present paper we apply the construction of Oseledec, mentioned above, to a rank 1 mixing transformation. With sufficient care, the resulting transformation is mixing (or even mixing of all orders), and admits an estimate of $M_{T}$. Our main result is the following:

THEOREM 1. There exists a mixing transformation $T$ with $2 \leq M_{T} \leq 6$. It is possible to insure that $T$ is mixing of all orders.

This theorem is proved in $\S 2$.

Using a straightforward generalization of the ideas in this paper and a lemma from [15], it is possible to obtain a more general result. Given an integer $m \geq 2$, let $p(m)$ be the smallest prime number such that $m$ divides $p(m)-1$ (the number $p(m)$ exists: [15]). 
THEOREM 2. There exists a mixing (or mixing of all orders) transformation $T$ such that $m \leq M_{T} \leq m p(m)$.

These results constitute a part of the author's 1983 University of Maryland Ph.D. dissertation [16], written under the supervision of Professor A. Katok. The author wishes to thank Professor Katok here for all of his help and encouragement. The present much simplified version of this paper was written at the MSRI in Berkeley, California.

This research was supported in part by NSF Grants MCS820404024/8102790.

\section{Proof of theorem 1}

Let $T_{0}$ be an invertible transformation of $[0,1 / 6]$, preserving normalized Lebesgue measure $\mu_{0}$. Denote by $\mathbb{Z} / m$ the cyclic group of order $m$, with additive notation and identified in the usual way with the set $\{0, \ldots, m-1\}$. Given a Borel function $\gamma:\left[0, \frac{1}{6}\right] \rightarrow \mathbb{Z} / 2$ we define $T_{1}$ on $\left[0, \frac{1}{6}\right] \times \mathbb{Z} / 2$ by

$$
T_{1}(x, y)=\left(T_{0} x, \gamma(z)+y\right) .
$$

The transformation $T_{1}$ is called the $\mathbb{Z} / 2$ extension of $T_{0}$ with cocycle $\gamma$. It preserves a natural probability measure which we denote by $\mu_{1}$.

The construction of $T_{1}$ from $T_{0}$ is a special case of a construction known as compact abelian group extension (cf. [10]). We apply this construction a second time. Let $\varphi: \mathbb{Z} / 2 \rightarrow \mathbb{Z} / 3$ be defined by $\varphi(0)=1$ and $\varphi(1)=2$. Define the transformation $T$ on $\left[0, \frac{1}{6}\right] \times \mathbb{Z} / 2 \times \mathbb{Z} / 3$ by

$$
T(x, y, z)=\left(T_{0} x, \gamma(x)+y, \varphi(y)+z\right) .
$$

Clearly, $T$ is a $\mathbb{Z} / 3$ extension of $T_{1}$. We denote the natural probability measure which $T$ preserves by $\mu$.

The transformation $T$ has a special property first discovered by Oseledec [13].

LEMMA 1. For any $T_{0}$ and $\gamma, M_{T} \geq 2$.

Proof (cf. [16]). There exists a $U_{T}$ invariant orthogonal decomposition

$$
L^{2}\left(\left[0, \frac{1}{6}\right] \times \mathbb{Z} / 2 \times \mathbb{Z} / 3, \mu\right)=H_{0} \oplus H_{1} \oplus H_{2},
$$

where

$$
H_{k}=\left\{\chi_{k}(z) f(x, y): f \in L^{2}\left(\left[0, \frac{1}{6}\right] \times \mathbb{Z} / 2, \mu_{1}\right)\right\},
$$

and

$$
\chi_{k}(z)=\exp 2 \pi i k z / 3 .
$$

It follows from a straightforward computation that $\left.V \circ U_{T}\right|_{H_{1}}=\left.U_{T}\right|_{H_{2}} \circ V$, where

$$
\left(V_{\chi_{1}} f\right)(x, y, z)=\chi_{2}(z) f(x, y+1)
$$

This symmetry implies that $M_{T} \geq 2$.

The next two lemmas will be helpful in establishing mixing for $T$.

LEMMA 2. If $T_{1}$ is weak mixing and $T$ is ergodic, then $T$ is weak mixing.

Proof. We note that $\left.U_{T}\right|_{H_{0}}$ is equivalent to $U_{T_{1}}$. If $T$ is not weak mixing then there is an eigenfunction $g$ for $U_{T}$ with eigenvalue $\lambda \neq 1$. Since $T_{1}$ is weak mixing, the 
projection of $g$ to $H_{0}$ is trivial. It follows that $g$ has a non-trivial projection $g^{\prime}$ in either $H_{1}$ or $H_{2}$ which is also an eigenvector for $\lambda$. Suppose $g^{\prime} \in H_{1}$. Then $V g^{\prime} \in H_{2}$ is another eigenfunction with eigenvalue $\lambda$. This implies that $g^{\prime} / V^{\prime}$ is a non-constant invariant function, contradicting the ergodicity of $T$.

Given $\gamma$, let $\bar{\gamma}=\gamma-1$ and let $\bar{T}_{1}$ denote the transformation constructed using $\bar{\gamma}$ instead of $\gamma$ in the definition of $T_{1}$. The next lemma is a special case of a theorem of Jones and Parry [7].

LemMA 3. If $T_{0}$ is weak mixing and both $T_{1}$ and $\bar{T}_{1}$ are egodic, then both $T_{1}$ and $\bar{T}_{1}$ are weak mixing.

Proof. Consider the $U_{T_{1}}$ invariant orthogonal decomposition

$$
L^{2}\left(\left[0, \frac{1}{6}\right] \times \mathbb{Z} / 2, \mu_{1}\right)=H^{0} \oplus H^{1},
$$

where $H^{k}=\left\{\chi_{k}(y) f(x): f \in L^{2}\left(\left[0, \frac{1}{6}\right], \mu_{0}\right)\right\}$ and $\chi_{k}(y)=\exp \pi i k y$. Here, $\left.U_{T_{1}}\right|_{H^{0}}$ is equivalent to $U_{T_{0}}$. Thus, given any eigenfunction $g$ with eigenvalue $\lambda \neq 1$, the projection of $g$ to $H^{0}$ is trivial, and consequently $g(x, y)=\chi_{1}(y) f(x)$. This implies $\chi_{1}(\gamma(x)) f\left(T_{0} x\right)=\lambda f(x)$ and $f^{2}\left(T_{0} x\right)=\lambda^{2} f^{2}(x)$.

Since $T_{0}$ is weak mixing, $\lambda^{2}=1$ and $f^{2}(x)=c=$ constant $\mu_{0}$-a.e. The ergodicity of $T_{1}$ rules out $\lambda=1$. On the other hand, if $\lambda=-1$, let $\psi$ be a $\mathbb{Z} / 2$-valued measurable function such that $C^{-1} f(x)=\exp 2 \pi i \psi(x)$. Then the eigenvalue equation for $g$ implies that

$$
\gamma(x)+\psi\left(T_{0} x\right)=1+\psi(x)
$$

or, in other words,

$$
\bar{\gamma}(x)=\psi(x)-\psi\left(T_{0} x\right) .
$$

One can easily verify that this contradicts the ergodicity of $\bar{T}_{1}$.

Now we will show how to construct $T_{0}, T_{1}$ and $T$. Note that by definition, $T_{1}$ and $T$ will be determined by $T_{0}$ and $\gamma$. In fact, we will construct all three of these transformations simultaneously by cutting and stacking. We refer to [5] for the details of this method of construction.

Recall that a transformation $T$ is said to be rank $r$ (cf. [8]) if there exists a cutting and stacking construction for $T$ with $r$ towers at each step, and no construction with fewer towers. Such a construction will be called a rank $r$ construction. A special case of a rank $r$ construction is what we will call a homogeneous rank $r$ construction. It may be described as follows:

After construction step $n$ we assume that there are $r n$-towers $\mathscr{Y}_{1}^{n}, \ldots, \mathscr{Y}_{r}^{n}$, of equal width and equal height. In step $n+1$, each tower $\mathscr{Y}_{k}^{n}$ is cut into $p_{n}$ columns $\mathscr{Y}_{k, 1}^{n} \ldots \mathscr{Y}_{k, p_{n}}^{n}$, of equal width. Then, for $k=1, \ldots, r$ and $j \leq p_{n}$, we stack $t_{n, j} \geq 0$ spacers on top of the column $\mathscr{Y}_{k, j}^{n}$ to obtain an augmented column $\overline{\mathscr{Y}}_{k, j}^{n}$.

Next we describe the stacking. For each $j<p_{n}$ let $\sigma_{j}^{n}$ be a given permutation of the set $\{1, \ldots, r\}$. Then, for each $k=1, \ldots, r$ and $j<p_{n}$, we stack the column $\overline{\mathscr{Y}}_{\sigma_{j}(k), j+1}^{n_{n}}$ on top of the column $\overline{\mathscr{Y}}_{k, j}^{n}$. In this manner we obtain $r$ new towers, which we number in such a way that the base of $\mathscr{Y}_{k}^{n+1}$ is a subset of the base of $\mathscr{Y}_{k}^{n}$. This completes step $(n+1)$. 
Of course, the numbers $p_{n}$ and $t_{n, j}$ must be chosen in such a way that the total measure is finite (cf. [12]). This being the case, the resulting transformation $T$ defined by the construction has a certain rank 1 transformation as a factor (i.e. the transformation constructed with a single tower at each step, using $p_{n}$ and $t_{n, j}$ ). It is well known that rank 1 transformations are ergodic (cf. [5]). We will now give a sufficient condition for the ergodicity of the transformation obtained from a homogeneous rank $r$ construction, $r>1$.

Let $k, l=1, \ldots, r$ and define

$$
g_{k, l}^{n}=\left(p_{n}-1\right)^{-1} \#\left\{j<p_{n}: \sigma_{j}^{n}(k)=l\right\} .
$$

Let $G^{n}$ be the $r \times r$ matrix with entries $g_{k, l}^{n}$. For $J \subseteq\{1, \ldots, r\}$, let $J^{c}$ denote the complement of $J$. Assuming $J, J^{c} \neq \varnothing$, let us define

$$
\begin{gathered}
E_{J}\left(G^{n}\right)=\sum_{\substack{k \in J^{c} \\
l \in J^{c}}} g_{k, l}^{n}, \\
E\left(G^{n}\right)=\min _{J, J^{c} \neq \varnothing} E_{J}\left(G^{n}\right) .
\end{gathered}
$$

We will say that $G^{n}$ is $\delta$-ergodic if $E\left(G^{n}\right)>\delta>0$.

The $\delta$-ergodicity of $G^{n}$ can be interpreted in terms of stacking during step $n+1$. Namely, if the $n$-towers are divided into any two collections, then a fraction at least $\delta$ of the columns from one collection will be stacked on top of columns for the other during stacking in step $n+1$.

It follows from a standard argument (cf. [8] or [16]) that if $G^{n}$ is $\delta$-ergodic for infinitely many $n$, then the transformation $T$ obtained from the construction is ergodic.

In the case $r=2$ these considerations are particularly simple. Suppose $T_{0}$ is rank 1 and let $\mathscr{Y}^{n}$ be the $n$-tower for $T_{0}$. Let us define $\tilde{\mathscr{Y}}^{n}$ to be the tower obtained from $\mathscr{Y}^{n}$ by removing the top level. A $\mathbb{Z} / 2$-valued Borel function $\gamma$ will be called rank 1 if for each $n$ it is constant on every level of $\tilde{y}^{n}$. One can easily see that in this case the transformation $T_{1}$ constructed from $T_{0}$ and $\gamma$ will admit a homogeneous rank 2 construction. Furthermore, every homogeneous rank 2 construction is of this type.

Let us define $\gamma_{n}=\gamma \mid{\tilde{\aleph_{n}+1}}_{\tilde{\vartheta}_{n}}$. The functions $\gamma_{n}$ have disjoint support (in the obvious sense) and $\gamma=\sum_{n=2}^{\infty} \gamma_{n}$. Furthermore, $\gamma$ is rank 1 if and only if $\gamma_{n}$ is rank 1 for each $n$. For a rank $1 \gamma, \gamma_{n}$ determines how the columns from the two $n$-towers are exchanged in building the $(n+1)$-towers during the homogeneous rank 2 construction for $T_{1}$. In particular, the $2 \times 2$ matrix $G^{n}$ is determined by $\gamma_{n}$, and it can always be made 1-ergodic by an appropriate choice of $\gamma_{n}$. It follows that for any rank $1 T_{0}$ there is a rank $1 \gamma$ so that $T_{1}$ is ergodic.

Let $N$ be a set of positive integers and let $\gamma^{N}=\sum_{n \in N} \gamma_{n}$. Observing that $\bar{\gamma}$ is rank 1 if and only if $\gamma$ is, we are led to the next lemma.

Leмma 4. Suppose $T_{0}$ is rank 1 and weak mixing. Let $N$ be an arbitrary infinite set of positive integers. Then there exists a choice of a rank $1 \gamma^{N}$ such that for any choice of rank $1 \gamma^{N^{c}}$, if $\gamma=\gamma^{N}+\gamma^{N^{c}}$ then the $\mathbb{Z} / 2$ extension $T_{1}$ of $T_{0}$ with cocycle $\gamma$ is weak mixing. 
Proof. Since $N$ is infinite, it contains two disjoint infinite subsets $N_{1}$ and $N_{2}$. The steps $n \in N_{1}$ are used to make $T_{1}$ ergodic and the steps $n \in N_{2}$ are used to make $\bar{T}_{1}$ ergodic. An application of lemma 3 implies that $T_{1}$ is weak mixing.

In a similar way, if $\gamma$ is rank 1 then $T$ will admit a homogeneous rank 6 construction. (We will consider the details of this construction in the proof of lemma 7 below.) It follows that in this case, the rank of $T$ is at most 6. Chacon [4] has proved that for any rank $r$ transformation $T, M_{T} \leq r$. This provides the desired estimate for $M_{T}$ :

LEMMA 5. If $T_{0}$ and $\gamma$ are rank 1 , then $2 \leq M_{T} \leq 6$.

Our next task is to show that this construction can be carried out so that $T$ is mixing. We observe that since $T_{0}$ is a factor of $T, T$ cannot be mixing (or mixing of all orders) unless $T_{0}$ is. D. Ornstein [12] has shown that rank 1 mixing transformations exist, and D. Rudolph [19] has generalized the argument to show that there exist rank 1 transformations which are mixing of all orders. From now on we will assume that $T_{0}$ is of this type.

Remark. It turns out that this assumption probably entails no loss of generality. S. Kalikow [9] has shown that any rank 1 mixing transformation (2-fold mixing in Kalikow's terminology) is actually 3 -fold mixing. It is likely that the proof can be strengthened to obtain mixing of all orders.

The next lemma was proved by J. P. Thouvenot (unpublished) for $k=2$ and D. Rudolph [20] for $k>2$. It is used to 'lift' the mixing property.

LEMMA 6. A weak mixing compact abelian group extension of a $k$-fold mixing transformation is $k$-fold mixing.

Note. We are using the terminology wherein 2-fold mixing is identical to mixing.

Remark. Similar lemmas are true for some stronger mixing properties: the $K$-property [14] and the Bernoulli property [18].

The final step in the proof of theorem 1 is the following lemma:

LEMMA 7. There exists an infinite set $M$ of positive integers with the following property. For any infinite subset $M_{1}$ of $M$ there exists a choice of a rank $1 \gamma^{M_{1}}$ such that for any choice of rank $1 \gamma^{M_{1}^{c}}$ the transformation $T$ constructed from $T_{0}$ and $\gamma=\gamma^{M_{1}}+\gamma^{M_{1}^{c}}$ is ergodic.

Proof. Note that $T_{0}$ and $T$ share the parameters $p_{n}$ and $t_{n, j}$. Let us define

$$
k_{n}=\#\left\{j<p_{n}: t_{n, j}>0\right\} \text {. }
$$

Since $T_{0}$ is assumed to be mixing, lim $\sup _{n \rightarrow \infty} k_{n} /\left(p_{n}-1\right)=2 \theta>0$, and $p_{n} \rightarrow \infty$ as $n \rightarrow \infty$ (i.e. there are both necessary conditions for a rank 1 transformation to be mixing). We define

$$
M=\left\{n: k_{n}>\theta\left(p_{n}-1\right)>12\right\}
$$

and note that $M$ is infinite.

We will show that if $n \in M$, there exists a rank $1 \gamma_{n}$ such that the $6 \times 6$ matrix $G^{n}$ for $T$ is $\delta$-ergodic. We begin with the following observation. Let $\alpha$ and $\beta$ be the 
permutations of $\{1, \ldots, 6\}$ defined by $\alpha(k)=k+3(\bmod 6)$, (where 0 and 6 are identified), and

$$
\beta(k)= \begin{cases}k+1 & \text { if } k \text { is odd } \\ k-1 & \text { if } k \text { is even. }\end{cases}
$$

Let $a_{n}=\#\left\{j<p_{n}: \sigma_{j}^{n}=\alpha\right.$ or $\left.\alpha^{2}\right\}$ and $b_{n}=\#\left\{j<p_{n}: \sigma_{j}^{n}=\beta \alpha\right.$ or $\left.\beta \alpha^{2}\right\}$. Then $G^{n}$ is $\delta$-ergodic for any $\delta \leq\left(p_{n}-1\right)^{-1} \min \left(a_{n}, b_{n}\right)$. This can easily be seen by identifying $G^{n}$ with a graph and observing how $\alpha, \alpha^{2}, \beta \alpha$ and $\beta \alpha^{2}$ contribute to the edge weights.

Now for $w=(x, y, z) \in\left[0, \frac{1}{6}\right] \times \mathbb{Z} / 2 \times \mathbb{Z} / 3$, let $\pi_{1}(w)=x, \pi_{2}(w)=(y, z)$ and $\tilde{\pi}(w)=y+2 z+1$. Let $L_{1}$ and $L_{2}$ be any pair of levels of $\overline{\mathscr{Y}}_{k_{j}}^{n}$ such that $T L_{1}=L_{2}$. As long as $L_{2}$ is not the top level of $\overline{\mathscr{Y}}_{k, p_{n}}^{n}, \pi_{1} L_{1}$ and $\pi_{1} L_{2}$ are levels of $\tilde{\mathscr{Y}}^{n}$ in the construction of $T_{0}$. Because it is rank $1, \gamma$ is constant on each of them. It follows from the definition of $T$ that for $w=(x, y, z) \in L_{1}$, if $c=\gamma\left(\pi_{1} L_{1}\right)$, then $\pi_{2}(T w)=(c+y, \varphi(y)+z)$. Moreover,

$$
\tilde{\pi}(T w)= \begin{cases}\alpha(\tilde{\pi} w) & \text { if } c=0 \\ \beta \alpha(\tilde{\pi} w) & \text { if } c=1\end{cases}
$$

In other words, if $\gamma=0$ on the top level of $\overline{\mathscr{Y}}_{k, j}^{n}$, we stack $\overline{\mathscr{Y}}_{k+1, \alpha(j)}^{n}$ on top of it. If $\gamma=1$ on the top level of $\overline{\mathscr{Y}}_{k j}^{n}$, we stack $\overline{\mathscr{Y}}_{k+1, \beta \alpha(j)}^{n}$ on top of it.

By induction, moving up the columns, one finds that each permutation $\sigma_{n}^{j}, j<p_{n}$, belongs to the group generated by $\alpha$ and $\beta$. Since $\alpha$ and $\beta$ satisfy the relations $\alpha^{2}=\beta^{3}=\alpha \beta \alpha \beta=1$, this group is isomorphic to the symmetric group $S_{3}$.

Now for $j<p_{n}$, let $L_{1}, L_{2}$, and $L_{3}$ be the top three levels of $\bar{y}_{k, j}^{n}, T^{2} L_{1}=T L_{2}=L_{3}$, and assume $t_{n, j}>0$. Then $\pi_{1} L_{2}$ and $\pi_{1} L_{3}$ are in the support of $\gamma_{n}$, and $\sigma_{j}^{n}=\omega_{1} \omega_{2} \omega_{3}$ where

$$
\begin{aligned}
& \omega_{1}=\left\{\begin{aligned}
\alpha & \text { if } \gamma_{n}=0 \text { on } \pi_{1} L_{3} \\
\beta \alpha & \text { if } \gamma_{n}=1 \text { on } \pi_{1} L_{3},
\end{aligned}\right. \\
& \omega_{2}=\left\{\begin{aligned}
\alpha & \text { if } \gamma_{n}=0 \text { on } \pi_{1} L_{2} \\
\beta \alpha & \text { if } \gamma_{n}=1 \text { on } \pi_{1} L_{2},
\end{aligned}\right.
\end{aligned}
$$

and $\omega_{3} \in S_{3}$. It follows that by varying the value of $\gamma_{n}$ on $\pi_{1} L_{3}$ and $\pi_{1} L_{2}, \sigma_{j}^{n}$ can be made equal to any of : $\omega_{1} \omega_{2} \omega_{3}, \beta \omega_{1} \omega_{2} \omega_{3}, \omega_{1} \beta \omega_{2} \omega_{3}$ or $\beta \omega_{1} \beta \omega_{2} \omega_{3}$. It is easy to check that one of these is always $\alpha$ or $\alpha^{2}$ and one is always $\beta \alpha$ or $\beta \alpha^{2}$.

We have assumed for this that $t_{n, j}>0$. If $n \in M$, this happens $k_{n}$ times, so that for an appropriate choice of $\gamma_{n}, a_{n}+b_{n}=k_{n}$ and $\left|a_{n}-b_{n}\right| \leq 1$. Thus,

$$
a_{n}, b_{n}>k_{n} / 3>\frac{\theta}{3}\left(p_{n}-1\right)>4
$$

and

$$
\left(p_{n}-1\right)^{-1} \min \left(a_{n}, b_{n}\right)>\theta / 3 .
$$

This implies that $G^{n}$ is $\theta / 3$ ergodic.

\section{Discussion}

The weak mixing results in [15] together with the results in this paper make the following conjecture plausible. 
CONJECTURE. For any given $m$ there exists a mixing transformation $T$ with $M_{T}=m$.

The conjecture would follow, for example, from the existence of a transformation $T$ with simple Lebesgue spectrum. In that case one would have, $M_{T^{m}}=m$, where $T^{m}$ denotes the $m^{\prime}$ th iterate of $T$. Although a complete solution to the Lebesgue spectrum problem does not seem close at hand, Mathew and Nadkarni [11] have recently made some encouraging progress by constructing a mixed spectrum example with a Lebesgue component of multiplicity 2 in the spectrum.

To see why the mixing construction in this paper is not enough to prove the conjecture, it is informative to see how it can be modified to obtain a weak mixing example. The modification is interesting in its own right because it provides an explicit cutting and stacking construction for a weak mixing transformation with $M_{T}=2$. With suitable changes, one can also obtain arbitrary finite $M_{T}$. (The examples obtained are essentially the same as those in [15].)

Let $p_{n} \rightarrow \infty$ and define:

$$
t_{n, j}= \begin{cases}0 & \text { if } n \text { is even and } j<p_{n} \\ 1 & \text { otherwise. }\end{cases}
$$

The rank 1 transformation constructed with these parameters is weak mixing (cf. [3]). Furthermore, if we choose $\gamma$ rank 1 , and having the additional property that for infinitely many construction steps, most of the top of one of the two towers for $T_{1}$ is mapped into the base of the other, then it follows from [1] that $T_{1}$ is rank 1 . In a similar way, it is possible to guarantee simultaneously that $T$ is rank 2 . Applying the same arguments as in the mixing case, we can obtain a weak mixing $T$ with $M_{T}=2$.

Now we show that in the mixing case, $T$ cannot be rank 2. K. Berg [2] has proved that any rank 1 mixing transformation is prime. This implies that $T_{1}$ cannot be rank 1 since it has $T_{0}$ as a factor. Recent work by D. Ullman (U.C. Berkeley dissertation) shows that if a rank 2 mixing transformation has a factor, it is unique and is rank 1 mixing. Therefore, $T$ cannot be rank 2 and mixing. Of course this does not imply that $M_{T}>2$, but it suggests that the techniques needed to estimate $M_{T}$ more exactly in the mixing case are more difficult than simple rank considerations.

\section{REFERENCES}

[1] J. R. Baxter. A class of ergodic transformations having simple spectrum. Proc. Amer. Math. Soc. 27 (1971), 275-279.

[2] K. R. Berg. Mixing, cyclic approximation, and homomorphisms. Preprint, University of Maryland.

[3] R. V. Chacon, Approximation of transformations with continuous spectrum. Pacific J. Math. 2 (1969), 293-302.

[4] R. V. Chacon. Approximation and spectral multiplicity. Springer Lect. Notes in Math. 160 (1970), 18-22.

[5] N. A. Friedman. Introduction to Ergodic Theory. Van Nostrand Reinhold Co.: New York (1970).

[6] G. R. Goodson. On the spectral multiplicity of a class of finite rank transformations. Preprint, University of Capetown.

[7] R. Jones \& W. Parry. Compact abelian group extensions of dynamical systems, II. Comp. Math. 25 (1972), 135-147. 
[8] A. del Junco. A transformation with simple spectrum which is not rank one. Can J. Math. 29 (1977), 655-663.

[9] S. Kalikow. Two fold mixing implies three fold mixing for rank one transformations. Ergod. Th. \& Dynam. Sys. 4 (1984), 237-259.

[10] A. Katok. Constructions in ergodic theory. To appear in Progress in Mathematics, Birkhauser, Boston, Mass.

[11] J. Mathew \& M. G. Nadkarni. A measure preserving transformation whose spectrum has Lebesgue component of multiplicity two. Bull. London Math. Soc. 16 (1984), 402-406

[12] D. S. Ornstein. On the root problem in ergodic theory. In Proceedings of the Sixth Berkeley Symposium on Statistics and Probability, Vol. II, University of California Press (1967), 1-49.

[13] V. I. Oseledec. The spectrum of ergodic automorphisms. Dokl. Akad. Nauk SSSR 168 (1966), 776-779.

[14] W. Parry. Ergodic properties of affine transformations and flows on nilmanifolds. Amer. J. Math. 91 (1961), 757-771.

[15] E. A. Robinson. Ergodic measure preserving transformations with arbitrary finite spectral multiplicities. Inv. Math. 72 (1983), 299-314.

[16] E. A. Robinson. Ergodic measure preserving transformations with finite spectral multiplicities. Dissertation, University of Maryland, Aug. 1983.

[17] E. A. Robinson. Transformations with highly non-homogeneous spectrum of finite multiplicity. In preparation.

[18] D. J. Rudolph. Classifying the isometric extensions of a Bernoulli shift. J. D'Analyse Math. 34 (1978), 36-60.

[19] D. J. Rudolph. An example of a measure preserving transformation with minimal self-joinings and applications. J. D'Analyse Math. 35 (1979), 97-122.

[20] D. J. Rudolph. K-fold mixing lifts to weakly mixing isometric extensions. Preprint, University of Maryland. 